\title{
A comprehensive analysis of cotton VQ gene superfamily reveals their potential and extensive roles in regulating cotton abiotic stress
}

Pengyun Chen ${ }^{1}$, Fei wei ${ }^{1,2}$, Shuaishuai Cheng ${ }^{1,3}$, Liang Ma1, Hantao Wang ${ }^{1}$, Meng Zhang ${ }^{1}$, Guangzhi Mao ${ }^{1}$, Jianhua Lu', Pengbo Hao ${ }^{1,3}$, Adeel Ahmad', Lijiao Gu', Qiang Ma', Aimin Wu', Hengling Wei ${ }^{1 *}$ and Shuxun Yu ${ }^{1 *}$ (D)

\begin{abstract}
Background: Valine-glutamine $(V Q)$ motif-containing proteins play important roles in plant growth, development and abiotic stress response. For many plant species, the VQ genes have been identified and their functions have been described. However, little is known about the origin, evolution, and functions (and underlying mechanisms) of the VQ family genes in cotton.

Results: In this study, we comprehensively analyzed the characteristics of 268 VQ genes from four Gossypium genomes and found that the VQ proteins evolved into 10 clades, and each clade had a similar structural and conservative motif. The expansion of the VQ gene was mainly through segmental duplication, followed by dispersal. Expression analysis revealed that many GhVQs might play important roles in response to salt and drought stress, and GhVQ18 and GhVQ84 were highly expressed under PEG and salt stress. Further analysis showed that GhVQs were co-expressed with GhWRKY transcription factors (TFs), and microRNAs (miRNAs) could hybridize to their cis-regulatory elements.

Conclusions: The results in this study broaden our understanding of the VQ gene family in plants, and the analysis of the structure, conserved elements, and expression patterns of the VQs provide a solid foundation for exploring their specific functions in cotton responding to abiotic stresses. Our study provides significant insight into the potential functions of $V Q$ genes in cotton.
\end{abstract}

Keywords: Gossypium, Valine glutamine (VQ), Phylogenetic, Expression analysis

\section{Background}

The $V Q$ genes form a large gene family with important roles in growth, development and abiotic stress tolerance in plants [1-3]. The VQ proteins have a conserved VQ motif [F**hVQ*hTG (F, phenylalanine; *, any amino acid; $\mathrm{h}$, hydrophobic residue; V, valine; $\mathrm{Q}$, glutamine; $\mathrm{T}$,

\footnotetext{
*Correspondence: henglingwei@163.com; ysx195311@163.com

'State Key Laboratory of Cotton Biology, Institute of Cotton Research of Chinese Academy of Agricultural Sciences, Anyang 455000, Henan, China Full list of author information is available at the end of the article
}

tryptophan; G, glycine)] [4, 5] and interact with WRKY TFs via the conserved residues V and Q. In Arabidopsis thanliana, many $V Q$ genes have been reported to function in development and responses to abiotic stress. For example, AtVQ23 (sigma factor-binding protein 1, SIB1), and AtVQ16 (SIB2) were found to interact with AtWRKY33 to increase the resistance of Arabidopsis plants to Botrytis cinerea [6]. In another study, AtVQ16 and $A t V Q 23$ have also been proven could interact with AtWRKY57, and AtVQ16 and AtVQ23 can enhance the

(c) The Author(s). 2020 Open Access This article is licensed under a Creative Commons Attribution 4.0 International License, which permits use, sharing, adaptation, distribution and reproduction in any medium or format, as long as you give appropriate credit to the original author(s) and the source, provide a link to the Creative Commons licence, and indicate if changes were made. The images or other third party material in this article are included in the article's Creative Commons licence, unless indicated otherwise in a credit line to the material. If material is not included in the article's Creative Commons licence and your intended use is not permitted by statutory regulation or exceeds the permitted use, you will need to obtain permission directly from the copyright holder. To view a copy of this licence, visit http://creativecommons.org/licenses/by/4.0/. The Creative Commons Public Domain Dedication waiver (http://creativecommons.org/publicdomain/zero/1.0/) applies to the data made available in this article, unless otherwise stated in a credit line to the data. 
competitions on AtWRKY57 to AtWRKKY33 in regulating JASMONATE ZIM-DOMAIN1 (JAZ1) and JAZ5 [7]. Moreover, JASMONATE-ASSOCIATED VQ MOTIF GENE1 (JAV1/AtVQ22) has been addressed to be as a key negative regulator of the jasmonate signalling [8]. For instance, AtVQ09 acts as a repressor of AtWRKY08 factor to modulate salt tolerance in Arabidopsis [9]. Recently, MdVQ10 and MdVQ15 were also described to interact with MdWRKY52 to regulate pathogen defense and development in apple (Malus domestica Borkh) [10]. In addition, OsVQ7 interacts with OsWRKY24 and play roles in NO signaling contributing to the tolerance of various stresses and development in rice (Oryza sativa) [11].

$V Q s$ have also been reported to perform other functions. It has been demonstrated that AtVQ14 (HAIKU, IKU1) interacts with MINISEED3 (MINI3, AtWRKY10) to reduce the expression of $I K U 2$, affecting the seed size [1], and AtVQ20 regulates the male gametogenesis in Arabidopsis [12]. In addition, some $V Q s$ have been shown to interact with ETHYLENE RESPONSE FACTORs (ERF), mitogen-activated protein kinases $(M A P K s)$, and miRNAs in response to environmental stresses in plants [13, 14]. Otherwise, some abiotic stress-related genes have been isolated from Arabidopsis and other plants [15-19], including VQ TFs, WRKY TFs, and other TFs.

Cotton is an important, widely cultivated fiber and oil crop that is essential for the textile industry and provides nutrient-rich edible oil [20]. Various biotic and abiotic stresses, including pathogen infection, drought and salinity stresses, consistently and severely affect the formation of cotton production [21-23]. Therefore, it is very important to develop new cultivars with high resistance to biotic and abiotic stresses. Recently, $V Q$ family genes have been identified at genome-wide levels in several plants, including Arabidopsis (34 VQs) [24], soybean (Glycine max) (74 VQs) [25], rice (39 VQs) [26], maize (Zea mays) (61 VQs) [27], Chinese cabbage (Brassica rapa spp. pekinensis) (57 VQs) [28], apple (49VQs) [10], tea (Camellia sinensis) (25 VQs) [29], tomato (Solanum lycopersicum) (26 VQs) [30], sunflower (Helianthus annuus) (20 VQs) [31], tobacco (Nicotiana tabacum) (59 VQs) [19], Cicer arietinum (19 VQs) [32] and Medicago truncatula (32 VQs) [32]. However, the genomic information and genetic evolution relationships of $V Q s$ are not clear in Gossypium spp., and the expression patterns of these genes in different tissues and in responses to abiotic stresses remain unknown. The released for cultivated Gossypium spp. (G. hirsutum Linn., G. barbadense L., G. raimondii Ulbr and G. arboretum L.) genome sequences and their annotation have brought much convenience for thoroughly investigation of their genomics and genetic information [33-35]. In this study, using the annotations of four Gossypium genomes, we identified the $V Q s$ of these Gossypium species, performed the analyses of phylogeny, conserved structural motifs, wholegenome duplication (WGD) and functional interaction networks of the $V Q s$, and predicted the microRNA target profiles of the $V Q s$. The comprehensive analysis of the $V Q$ gene family in Gossypium will contribute to identify new key candidate genes for diverse stress resistance in cotton breeding.

\section{Results}

Identification and comparative analysis of VQs in plants To identify VQ family in G. hirsutum, G. barbadense, G. raimondii and $G$. arboretum, the AtVQ proteins were used as query sequences to search against the protein databases of the four Gossypium species, and the VQdomain Pfam (PF05678) was also applied. In total, 89 GhVQs, $89 \mathrm{GbVQs}, 45 \mathrm{GrVQs}$, and $45 \mathrm{GaVQs}$ were identified and named in G. hirsutum, G. barbadense, G. raimondii and $G$. arboretum, respectively (Additional File 1, Supplemental Table S1). In addition, the physiological and biochemical properties of $268 \mathrm{VQs}$ in Gossypium species were determined, including CDS length, GC count, isoelectric point (pI) and molecular weight (MW) (Additional File 1, Supplemental Table $\mathrm{S} 1)$. The CDS lengths of these Gossypium $V Q s$ ranged from $279 \mathrm{bp}$ (GhVQ89 and GbVQ89) to $1443 \mathrm{bp}$ (GbVQ15), the average GC content of the transcripts was 46.01, their exon numbers varied from 1 to 9 , and only a small percentage of $V Q s$ contained introns $3.37 \%$ GhVQs, 3.37\% GbVQs, $6.67 \%$ GaVQs, and $31.11 \%$ GrVQs). The pI values varied from 4.159 (GbVQ33 and GbVQ78) to 11.496 (GhVQ07) and the MW values ranged from $10.346 \mathrm{kDa}$ (GbVQ89) to $52.058 \mathrm{kDa}$ (GbVQ15) (Additional File 2, Supplemental Fig. S1).

To perform comparative genomic analyses, we searched another 11 plant species for VQ proteins. The evolutionary relationships of the species and the number of their $V Q$ genes are shown in Fig. 1. The data revealed that the numbers of $V Q s$ in $A$. trichopoda, $P$. dactylifera, $V$. vinifera, $P$. trichocarpa, and $T$. cacao were less than those in the four Gossypium species (Additional File 3, Supplemental Table S2). The comparative structure analysis of $V Q s$ showed that almost all the $V Q s$ had a few introns and encoded relatively small proteins, and only 3 GhVQs, 3 GbVQs, 3 GaVQs and 14 GrVQs had more than one intron. We speculated that the WGD events that occurred during the evolution of angiosperms increased the numbers of the Gossypium VQs, and these events helped the $V Q s$ to gain new functions through neofunctionalization. However, the evolutionary forces that shaped the current intron/exon gene structures remain unknown. 

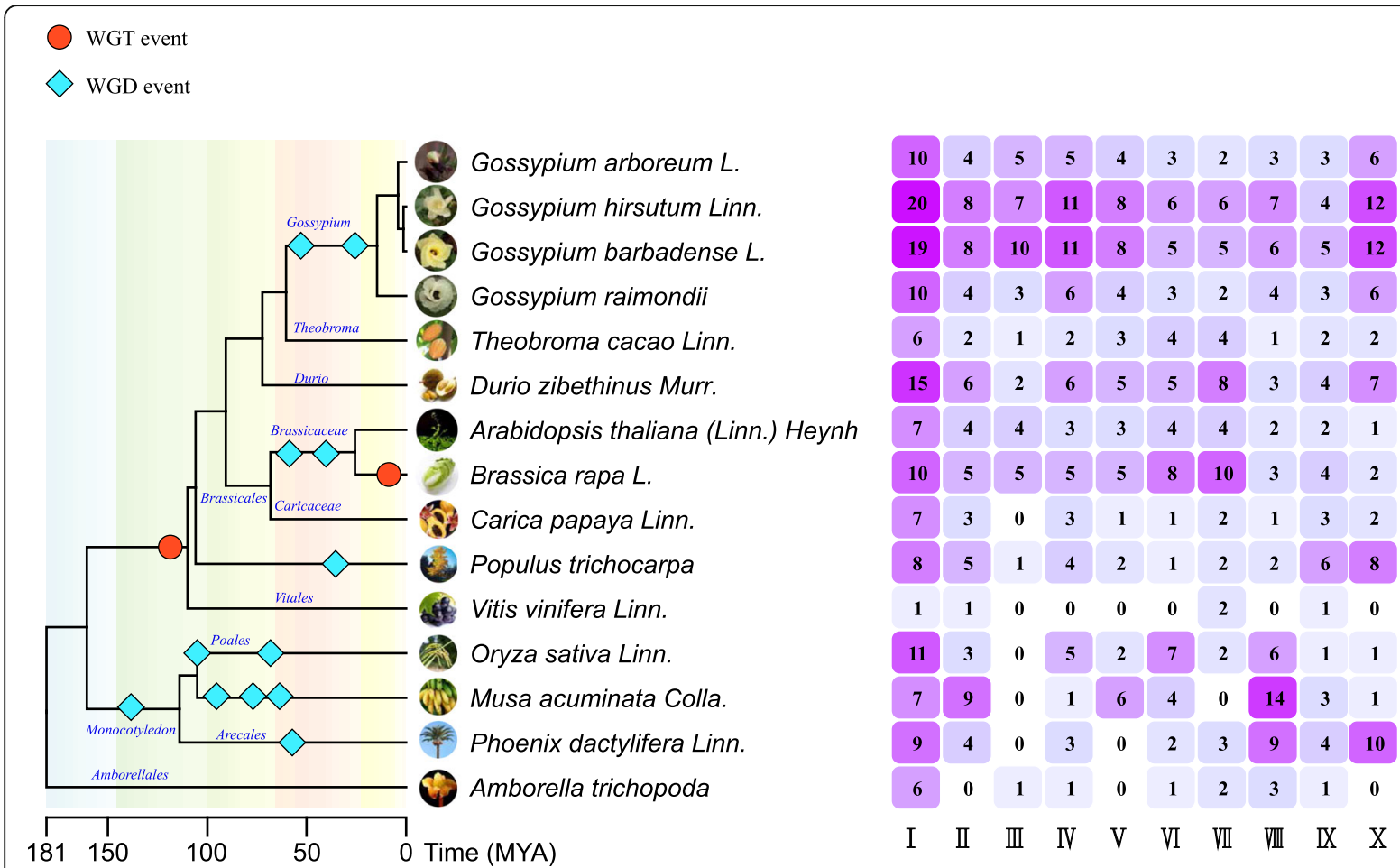

Fig. 1 The VQ gene family evolutionary relationship and the number details of the 15 plant species. The left of this figure shows the evolutionary relationships of the species; the right of this figure shows the number details of the VQ family of each group

\section{Phylogenetic analysis of VQs}

To explore the relationships among VQs in Gossypium, we conducted a phylogenetic analysis of the VQs from the 15 plant species (Fig. 2), and a phylogenetic tree between Gossypium spp. and Arabidopsis was also constructed (Additional File 4, Supplemental Fig. S2). The tree contained 656 VQs. These proteins were divided into 10 clades based on the nomenclature of the Arabidopsis VQs. The largest group (Group I) contained 20 GhVQs, 20 GbVQs, 10 GaVQs, and 10 GrVQs. Group IX was the smallest group, including 4 GhVQs, 5 GbVQs, 3 GaVQs and 3 GrVQs. Previous research has verified that VQ proteins contain a conserved motif composed of $F^{* * h}$ hVQ"hTG [4, 5]. In our study, among the 656 VQs, 212 proteins (in Group I and Group II) had the amino acid "M" next to "VQ" (simple M-VQ model); 159 proteins (in Group III, Group IV, and Group VII) had the amino acid "V" next to "VQ" (simple V-VQ model); and 285 proteins (in Group V, Group VI, Group VII, Group IX and Group X) had the amino acid "L" next to "VQ" (simple L-VQ model) (Additional File 5, Supplemental Fig. S3). The VQs with rarer amino acids of the Gossypium species were also scattered in Group I to Group X, and the clusters of VQs were similar to those in angiosperms [3].
Cis-regulation elements and structural composition of the VQs

The cis-regulation elements in the promoters (from $2000 \mathrm{bp}$ to $-1 \mathrm{bp}$ ) of the four Gossypium VQs were analyzed using the PlantCARE tool. 715, 701, 386 and 399 cis-regulation elements from the GhVQs, GbVQs, GaVQs and GrVQs were identified, respectively. Among these, seven kinds of hormone-responsive cisregulation elements, ABRE (ABA-responsive element), P-box, TGA-box, TGA-element, TCA-element, CGTCA-motif, and GARE-motif were associated with ABA, ethylene, salicylic acid (SA), methyl jasmonate (MeJA), auxin (IAA), and gibberellin (GA), respectively; and six regulatory elements, MBS, TC-rich repeats, LTR, DRE-motif, W-box, and CCAAT-box were related to drought, cold stress, and pathogen defense (Fig. 3). Moreover, the promoters of 66 GhVQs, 69 GbVQs, 36 GrVQs and 34 GaVQs possessed WRKY-binding sites (W-box) (Fig. 3). The diversity of the cis-regulation elements in the promoters of $V Q s$ indicated that $V Q s$ might participate in regulating the Gossypium response to endogenous hormones and diverse environmental stimuli.

Motif compositions and exon-intron structures of the $V Q s$ were shown in Fig. 4. Combining the phylogenetic data groups of the four Gossypium $V Q s$, we 


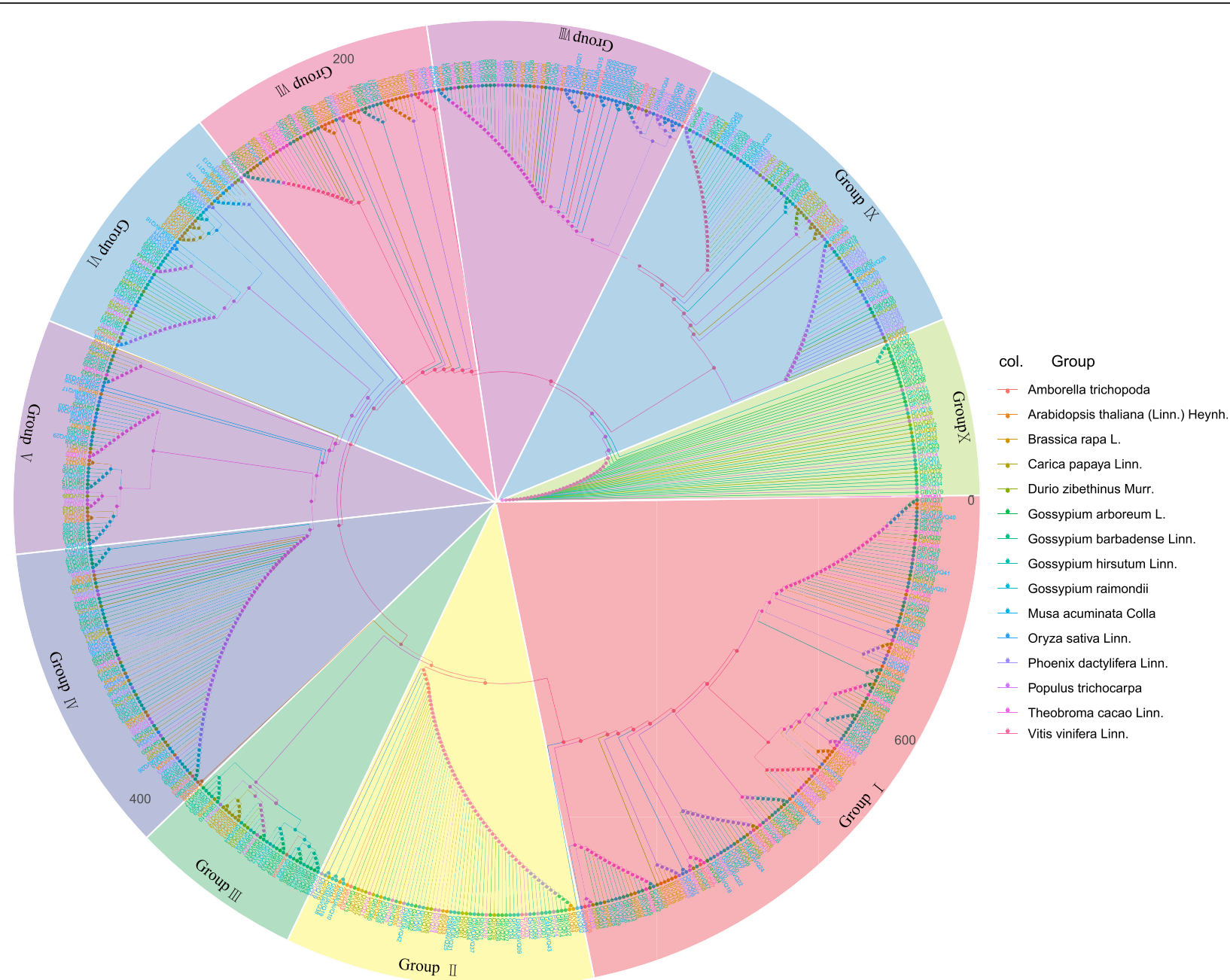

Fig. 2 The VQ gene family phylogenetic relationship of the 15 plant species. The Phylogenetic tree includes 268 VQs from Gossypium and 388 VQs from other 11 plants. Maximum likelihood (ML) bootstrap values are shown in the major nodes

found that there were more motif types in Group IV, including Motif 1, Motif 4, Motif 7, Motif 9, and Motif 10; and followed by Group II, containing Motif 1, Motif 2, Motif 3, Motif 6, and Motif 8 . Not surprisingly, Motif 1 existed in almost all of the $V Q s$, suggesting that it was the most conservative motif. The differences in motif composition among the four Gossypium VQs suggested that they might perform different functions in diverse Gossypium species. Most of the VQs had no intron, including $96.63 \%(86 / 89)$ of GhVQs, 96.63\% (86/89) of GbVQs, 68.89\% (31/45) of GrVQs, and $93.33 \%(42 / 45)$ of GaVQs. The remaining $V Q s$, which were widely distributed in Group VII and Group X, contained one to eight introns. In general, $V Q s$ in the same clades would share similar motif elements and structural compositions, indicating that the $V Q$ members in the same subgroup could have similar functions.

\section{Chromosomal distribution, synteny and duplication} analysis

In this study, $V Q s$ were detected located in most chromosomes with a few exceptions. For example, Gh A09 and Gh_D02 were in G. hirsutum, Gb_A09, Gb A13 and Gb_D02 were in G. barbadense, Ga_Chr03 and Ga_Chr09 in G. arboretum, and Gr_Chr05 were G. raimondii (Fig. 5). For the two allotetraploid species of Gossypium, Gh_D05 (eight genes/ 9\%), Gb_A05 (eight genes / 9\%), and Gb_D05 (eight genes / 9\%) contained more $V Q$ genes than other chromosomes, while Gh_A02, Gh_A03, Gh_A13, Gh_D08, Gh_D13, Gb_A02, Gb_A03, Gb_D08, Gb_D09, and Gb_D13 only contained one gene. For the two diploid species, Ga_Chr05 (eight genes / 17.8\%), Gr_Chr02 (seven genes / 17.8\%), Gr Chr07 (seven genes / 17.8\%) and Gr_Chr09 (seven genes / 17.8\%) contained more VQs, and Ga_Chr08, Ga_Chr13, Gr_Chr04, Gr_Chr12, and Gr_Chr13 only 


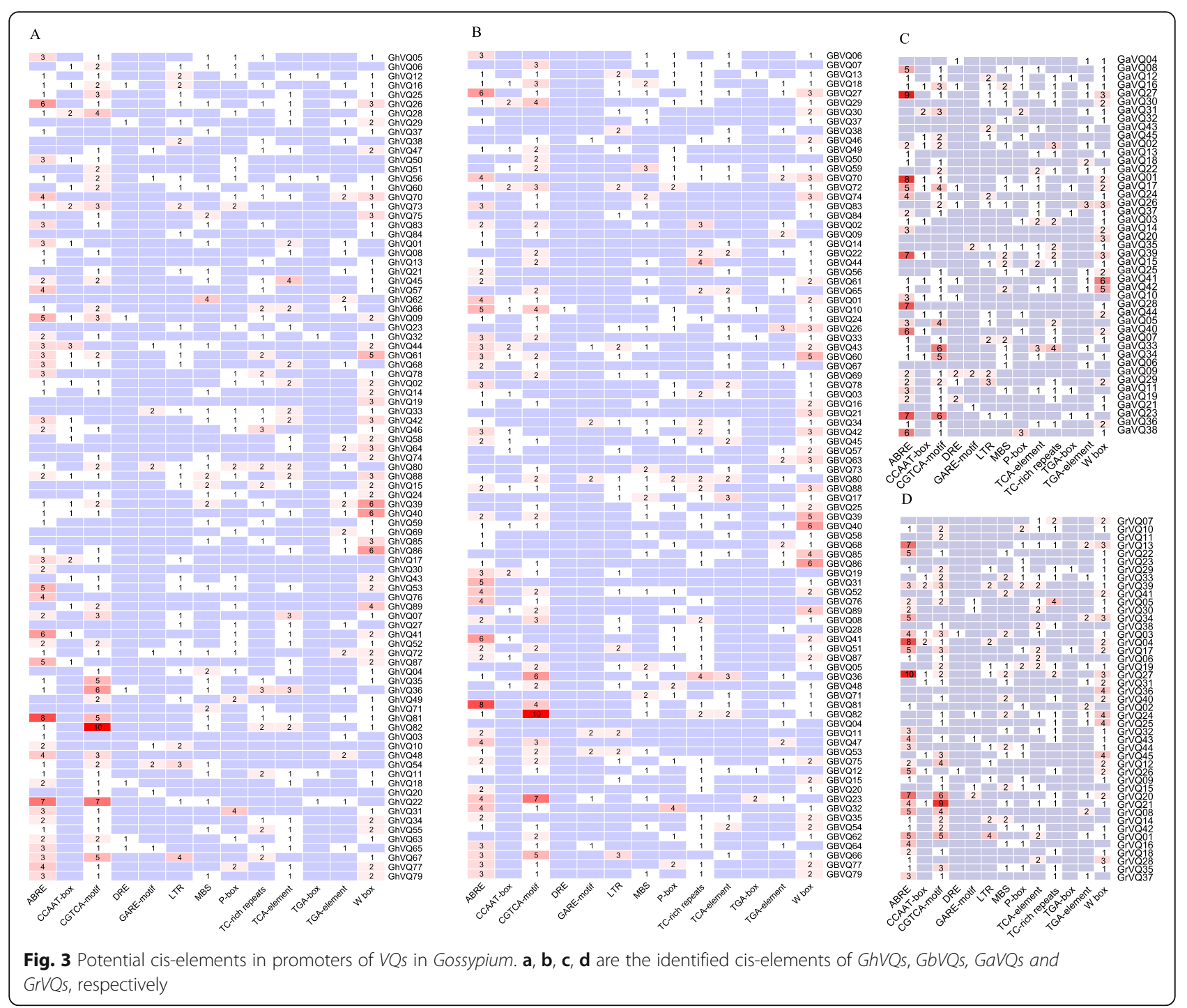

contained one gene. Most VQs in the four Gossypium species were distributed at both ends of the chromosomes, which corresponded to the position of the telomere.

The orthologous $V Q s$ were first identified between $G$. hirsutum and G. arboreum with G. raimondii. A total of 83 GhVQs were orthologous genes in the two-diploid Gossypium, of which 40 gene pairs showed A genome origin, while 43 gene pairs showed D genome origin (Additional Files 6 and 7, Supplemental Fig. S4 and S7, Table S3). Subsequently, orthologous gene identification was also conducted between G. barbadense and G. arboreum with G. raimondii, and there were 84 orthologous GbVQs. Of which, 40 gene pairs showed A genome origin, while 44 gene pairs showed $\mathrm{D}$ genome origin (Additional Files 7 and 8, Supplemental Fig. S5 and S3). Orthologous genes between G. hirsutum and G. barbadense were also identified. It was found that 39 gene pairs in Gh_At and Gb_At subgenomes and 42 gene pairs in Gh_Dt and Gb_Dt subgenomes (Fig. 6 and Additional File 7, Supplemental Table S3). In addition, GhVQ27, GhVQ61, GhVQ68, GbVQ28, GbVQ60 and GbVQ67 had no orthologous genes in the diploid Gossypium species.

As previously described, duplication contributed to the expansion of genes in the polyploid events in plants [36]. The tetraploid species Gossypium of have undergone a genome duplication since their diverge from the two diploid species of Gossypium. In our study, we have identified the $V Q$ duplication event, and the WGD/segmental event likely contributed to the expression regulation of $V Q s$ in Gossypium. The percentages of $V Q s$ derived from WGD were $60.47 \%$ in the At-subgenome of G. hirsutum, 63.04\% in the Dt-subgenome of G. hirsutum, $69.04 \%$ in the At-subgenome of G. barbadense, $59.57 \%$ in the Dt-subgenome of G. barbadense, $62.22 \%$ 


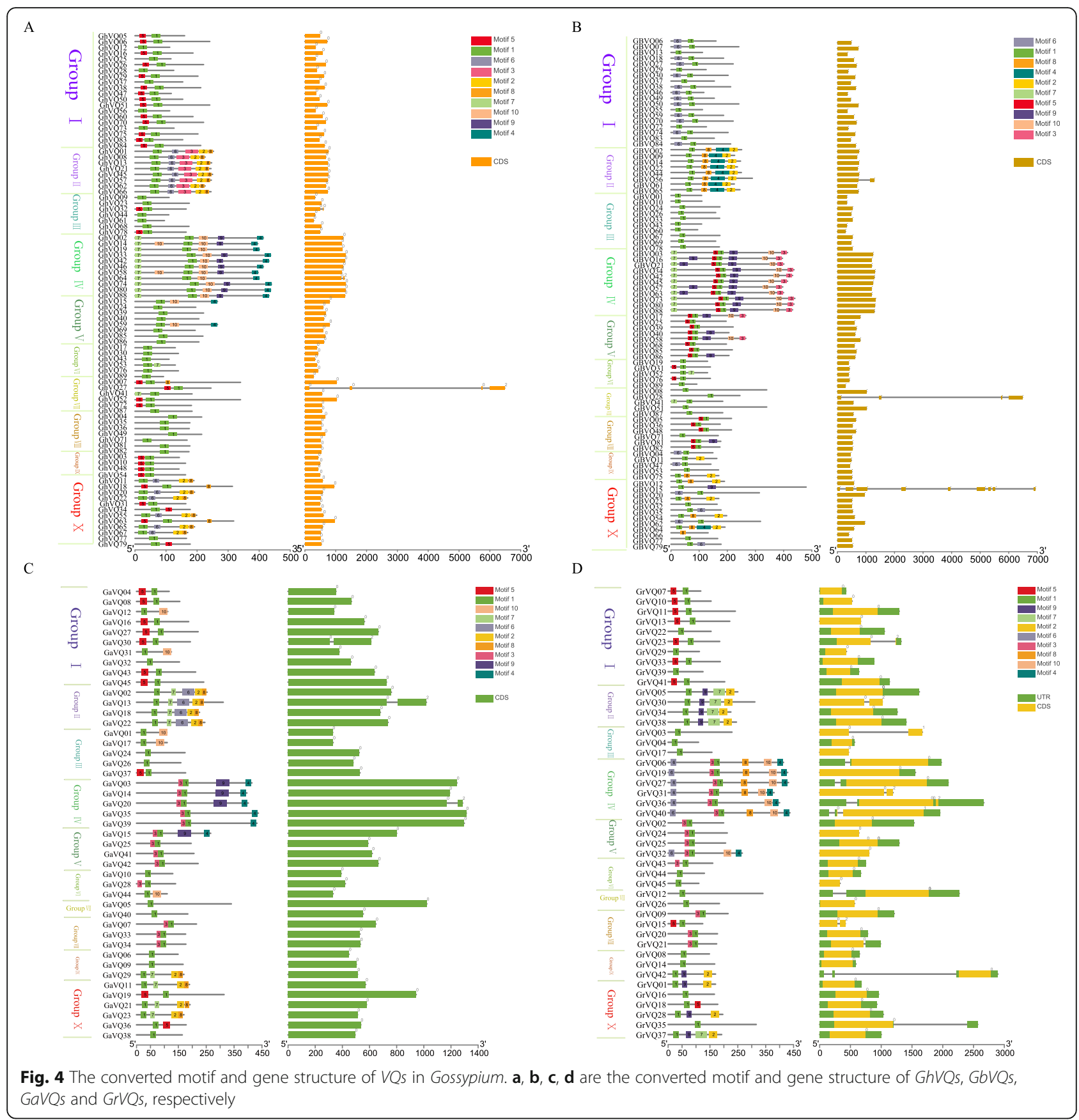

in G. raimondii, and $57.78 \%$ in G. arboretum (Additional File 9, Supplemental Table S4). Gene duplication events after the divergence of Gossypium species resulted in a high number of paralogous genes in both allotetraploid Gossypium species.

\section{Prediction of miRNA target sites}

miRNA had been predicted to target the $V Q$ genes in Arabidopsis [37, 38] and tea [39]. To determine the miRNA-mediated post-transcriptional regulation of $V Q s$ in two allotetraploid species of Gossypium, we predicted the target sites of miRNAs in the coding (CDS) regions of the GhVQs and GbVQs. In G. hirsutum, 46 sites of 34 GhVQs were detected that could be targeted by $22 \mathrm{miR}$ NAs, while 46 sites of 32 GbVQs could be targeted by 21 miRNAs (Fig. 6 and Additional File 10, Supplemental Table S5). Of these, six $V Q$ genes (GhVQ02, GhVQ40, GhVQ86, GbVQ02, GbVQ40 and GbVQ86) were predicted to be targeted by Ghr-miR172 in the CDS regions; and six VQ genes (GhVQ39, GhVQ52, GhVQ85, 


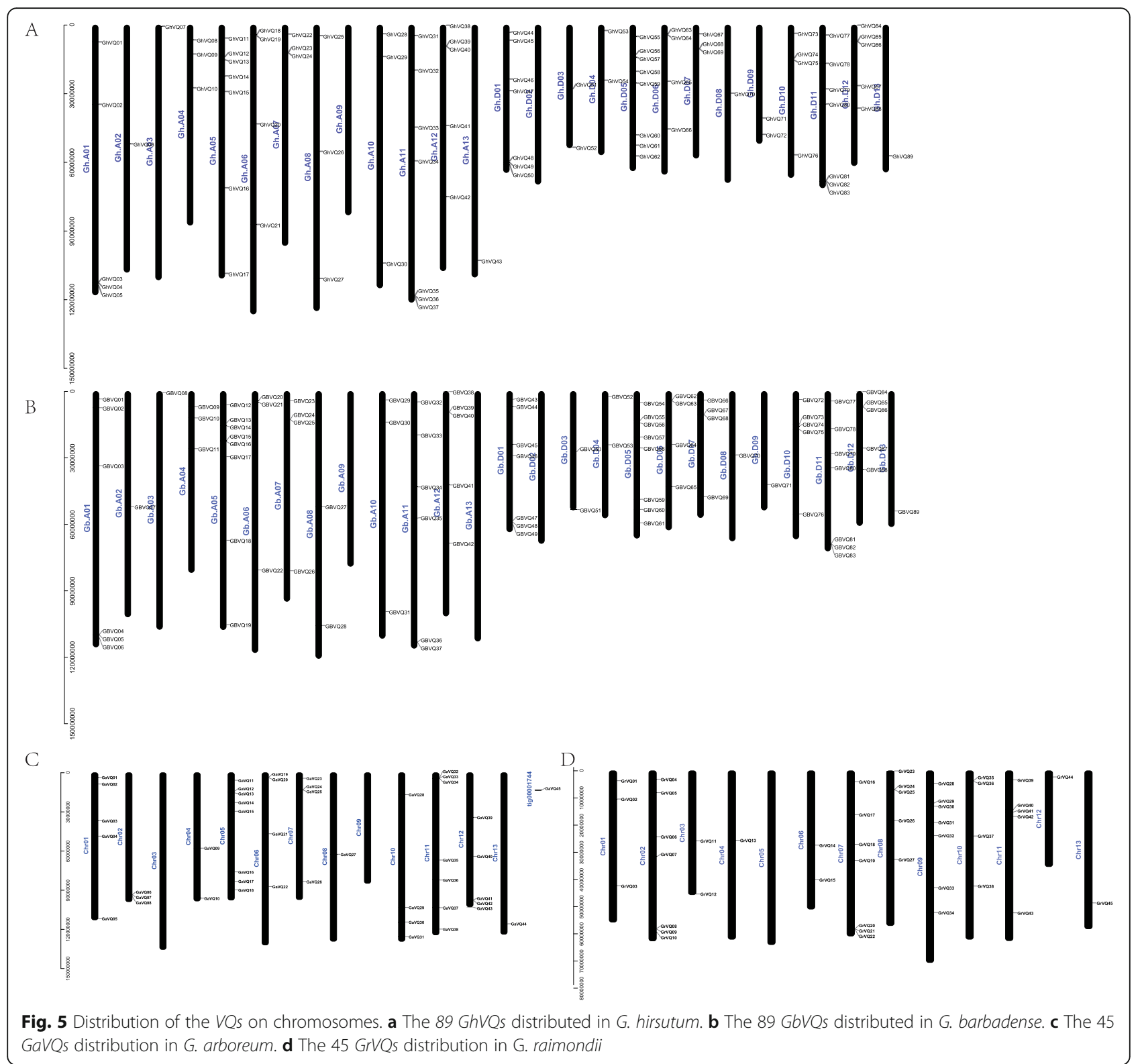

GbVQ39, GbVQ51 and GbVQ85) were targeted by GhrmiR156 (Ghr-miR156a, Ghr-miR156b, Ghr-miR156c and Ghr-miR156d) at 10 prediction sites. Ghr-miR172 and Ghr-miR156 were reported to be involved in some biological processes, including the responses to developmental cues and abiotic stress in plants [40-42]. However, it requires further experiments to verify the regulation mechanism and functions of those predicted miRNAs and their targets in Gossypium.

\section{Expression pattern analysis and function verification}

Expression profiles of the $V Q s$ in the two allotetraploid kinds of Gossypium were analyzed with available transcriptome data (Additional Files 11 and 12,
Supplemental Fig. S6 and S7 and Supplemental Table S6). In this study, the GhVQs and GbVQs with average FPKM values $>5$ and being present in at least two samples were identified as potentially expressed transcripts. Fifty-seven GhVQs and $39 \mathrm{GbVQs}$ were selected, and their expression profiles tested in 10 tissues, including the anther, bract, filament, leaf, petal, pistil, root, sepal, stem and torus (Fig. 7). For 57 GhVQs, 22 genes were highly expressed in roots and GhVQ82 had the highest expression level, and 14 genes were highly expressed in leaves. There were only a few genes expressed in the anther, bract, filament, petal, pistil, sepal, stem and torus (Fig. 7a). For 39 GbVQs, 17 GbVQs were strongly highly expressed in roots, and 12 genes were strongly expressed 


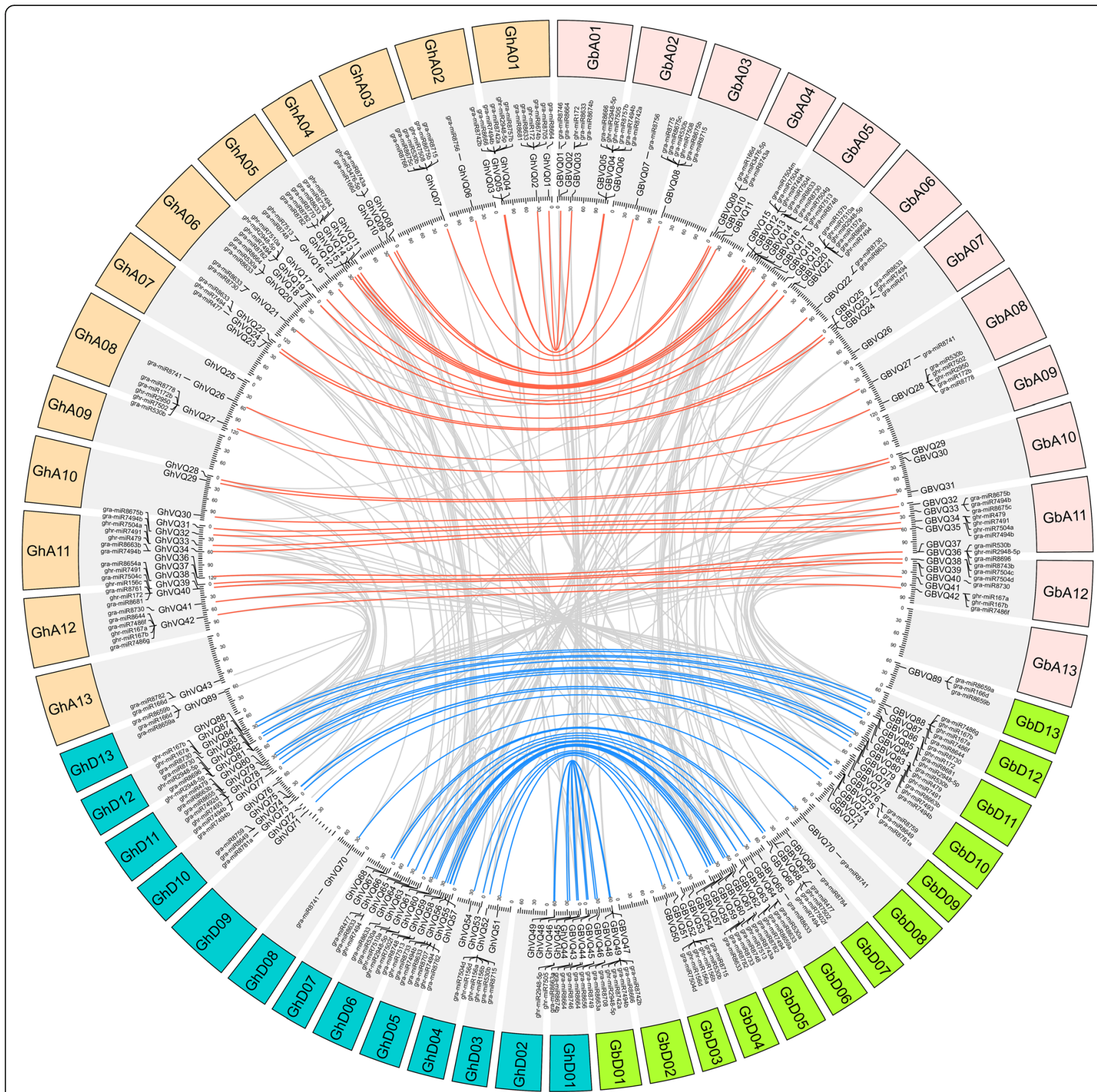

Fig. 6 Ortholog and paralog pairs of VQs in G. hirsutum and G. barbadense. The lines regarding orthologous gene pairs are colored by blue and orange, and the paralogous gene pairs are colored by grey lines. The central links are predicted miRNA target genes

in leaves (Fig. 7b). The different expression profiles of $V Q$ genes suggest that they have different functions in distinct tissues and developmental stages.

$V Q s$ were widely identified in the abiotic stress responses in angiosperms [3, 32]. In this study, the expression patterns of $V Q s$ in the allotetraploid Gossypium types under salt, drought, cold and heat stresses were analyzed using the published data. In total, $43 \mathrm{GhVQs}$ and $37 \mathrm{GbVQs}$ had different expression profiles under the four abiotic stress treatments (Fig. 8). Under salt stress, 29 GhVQs were significantly up-regulated at $12 \mathrm{~h}$, and $21 \mathrm{GbVQs}$ were up-regulated at $6 \mathrm{~h}$ (Fig. 8a and c). Upon PEG treatment, most of the GhVQs and GbVQs were highly expressed at $12 \mathrm{~h}$ (Fig. 8b and d). During cold stress, 33 GhVQs and 19 GbVQs were up-regulated at $24 \mathrm{~h}$ (Fig. 8e and g). Most of the GhVQs and GbVQs under the hot treatment were highly expressed at $1 \mathrm{~h}$ (Fig. 8f and h). To validate of the expression results of GhVQs in response to salt and drought stresses, we conducted qRT-PCR analyses of 12 GhVQs after treatments with PEG and salt treatment. In the presence of PEG, GhVQ08, GhVQ18, GhVQ62, GhVQ64, GhVQ80, and 


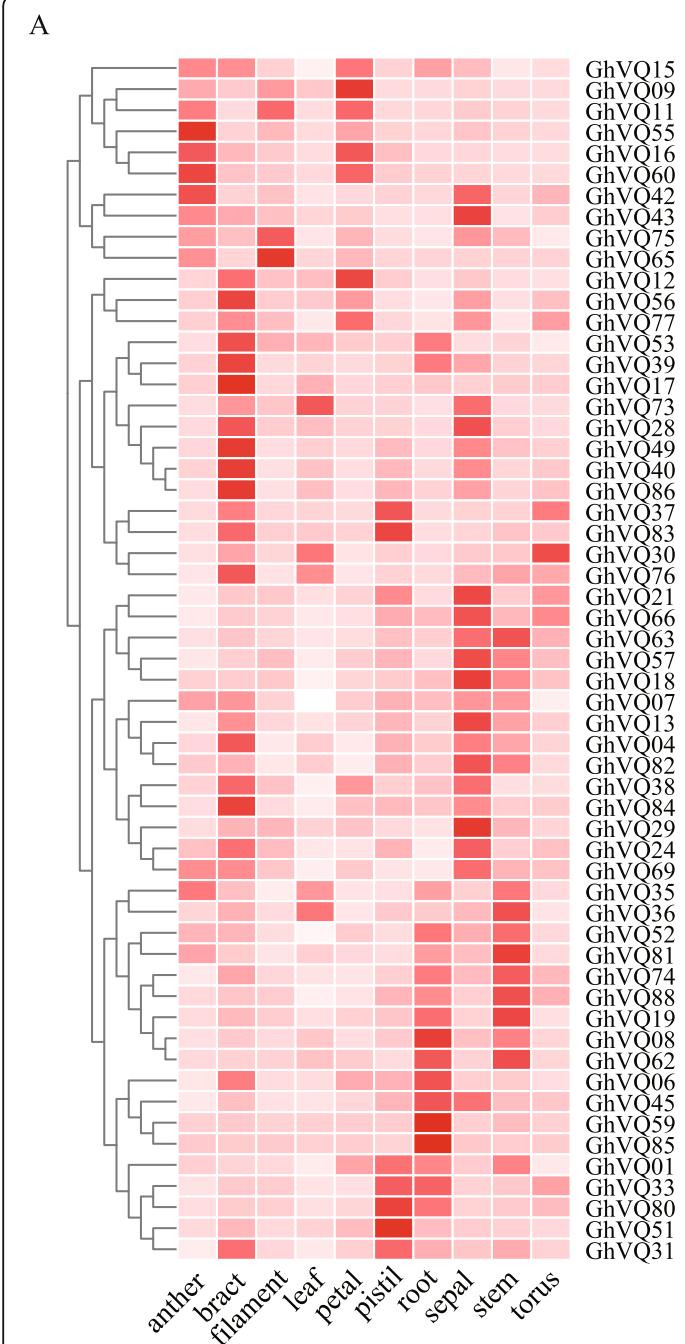

B

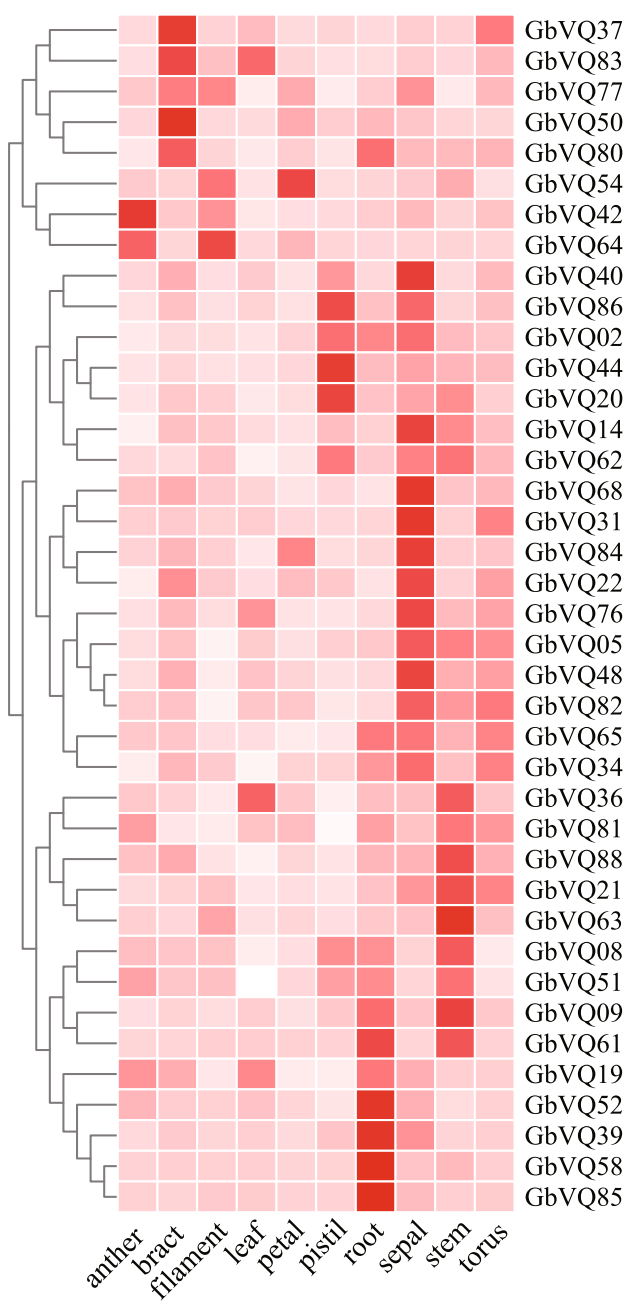

Fig. 7 The expression of Gossypium VQs in different tissues. a The expression of the selected VQs in G. hirsutum; b The expression of the selected VQs in G. barbadense

GhVQ84 had high expression levels at $48 \mathrm{~h}$, while these GhVQs except GhVQ18 and GhVQ84, were highly expressed during 24-48 h under salt treatment (Fig. 9). The qRT-PCR results were slightly different from the RNA-seq data, but these findings suggest that some GhVQs were involved in plant response to drought and salt stresses.

\section{Co-expression and interaction networks of GhVQs}

To understand the putative roles of $V Q s$ in plant adaptation to drought and salt stresses, we conducted a coexpression analysis. Ten GhVQs were found to coexpress with another 227 functional genes (Fig. 10 and Additional File 13, Supplemental Table S7). Among these, six and seven $V Q s$ were identified in different modules of drought stress and salt stress, respectively, while GhVQ37, GhVQ59 and GhVQ83 were detected coexisting during the two stress treatments. Moreover, these 227 genes co-expressing with $10 \mathrm{GhVQs}$, contained multiple TFs, including domain AP2, bHLH, F-box, GRAS, p450, PLB03212, WD40 and WRKY (Fig. 10a-e and Additional File 13, Supplemental Table S7). The functional regulation networks of the GhVQs were constructed using the website of STRING11.0 with the module reference of Arabidopsis association, and the results revealed that the GhVQs participated in plant defense interaction networks, including WRKYs, MYB15, MPK4, AR781, CSN5B and SIGAs (Fig. 10f). Indeed, VQ proteins could interact with WRKYs and other TFs to defend against abiotic stresses in cotton.

\section{Discussion}

In previous studies, the $V Q$ family genes have been systematically analyzed in Arabidopsis [24], soybean [25], sunflower [31], rice [26], banana [43], maize [27], bamboo [44], Cicer arietinum [32], Medicago truncatula 


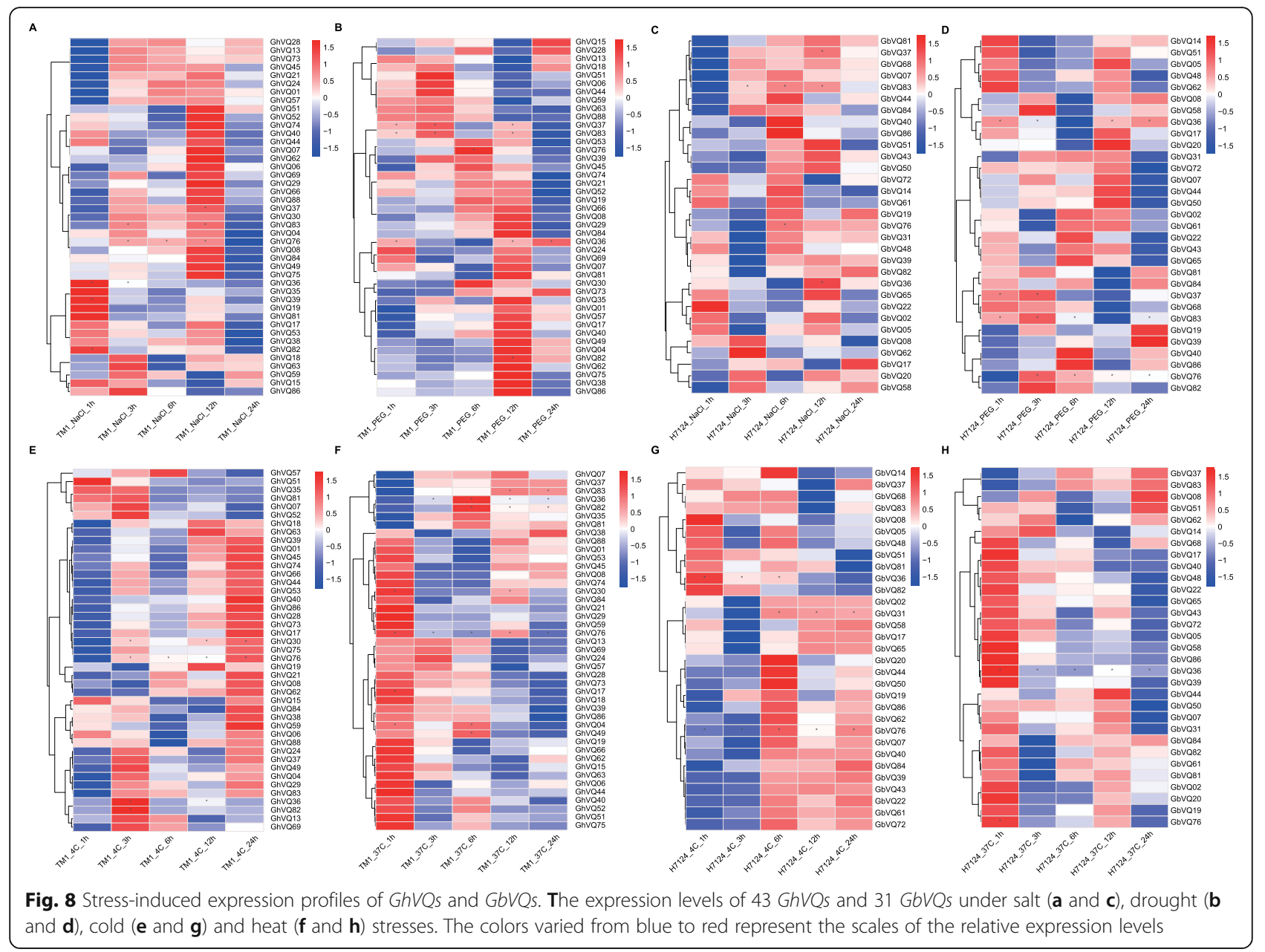

[32], and tobacco [19], and has shown to play significant roles in regulating growth, development processes, and responding to biotic and abiotic stresses [2]. Here, we complete a comprehensive analysis of the $V Q$ family genes and explore their evolutional mechanism in Gossypium species.

\section{The expansion, duplication and structural characteristics of VQs in Gossypium}

In this study, we analyzed the $V Q s$ of G. hirsutum, $G$. barbadense., G. raimondii, G. arboretum and another 11 plant species, and found that the number of $V Q s$ in the genomes of 15 species was inconsistent with related to the size of their genomes. There are $89 G h V Q s, 89$ $G b V Q s, 45 G a V Q s$, and $45 \mathrm{GrVQs}$, respectively. The number of Gossypium $V Q s$ was higher than that in cacao (27 TcVQs) and in Arabidopsis (34 AtVQs), but the number of GrVQs and GaVQs was fewer than the $D z V Q s$ (Fig. 1 and Additional File 2, Supplemental Table S2). Previous studies have shown that diverse WGD events lead to the different sizes of plant genomes [45-
47]. Our results indicated that $V Q s$ in these four Gossypium species were more likely to be proximal, tandem, and segmental genes, while the majority of $V Q s$ in rice [26] and Arabidopsis [24] are singleton genes. Through the analysis of the phylogenetic and structural features of the 15 plants VQ domains, the $V Q s$ could be divided into 10 clades. Group III could be expanded in the eudicots, particularly in the Mallow species (Fig. 2), while Groups III, IV, V, VI, VII, and X had no $V v V Q s$, suggesting that these might have been lost in ancient genome duplication events.

Ten conserved motifs were also identified in the four Gossypium VQs. Motif 1 corresponded with the VQcontaining motif, which is widely found in angiosperms [3]. Previous studies have suggested that $V Q s$ have few introns in higher plants, being agreement with the results from Gossypium VQs. Only 3 GhVQs, 3 GbVQs, 14 $\mathrm{GrVQs}$, and $3 \mathrm{GaVQs}$ had multiple introns. Additionally, the motif compositions and intron contents of the Gossypium VQ proteins/genes in our study were consistent with the results of the phylogenetic analysis and the type 


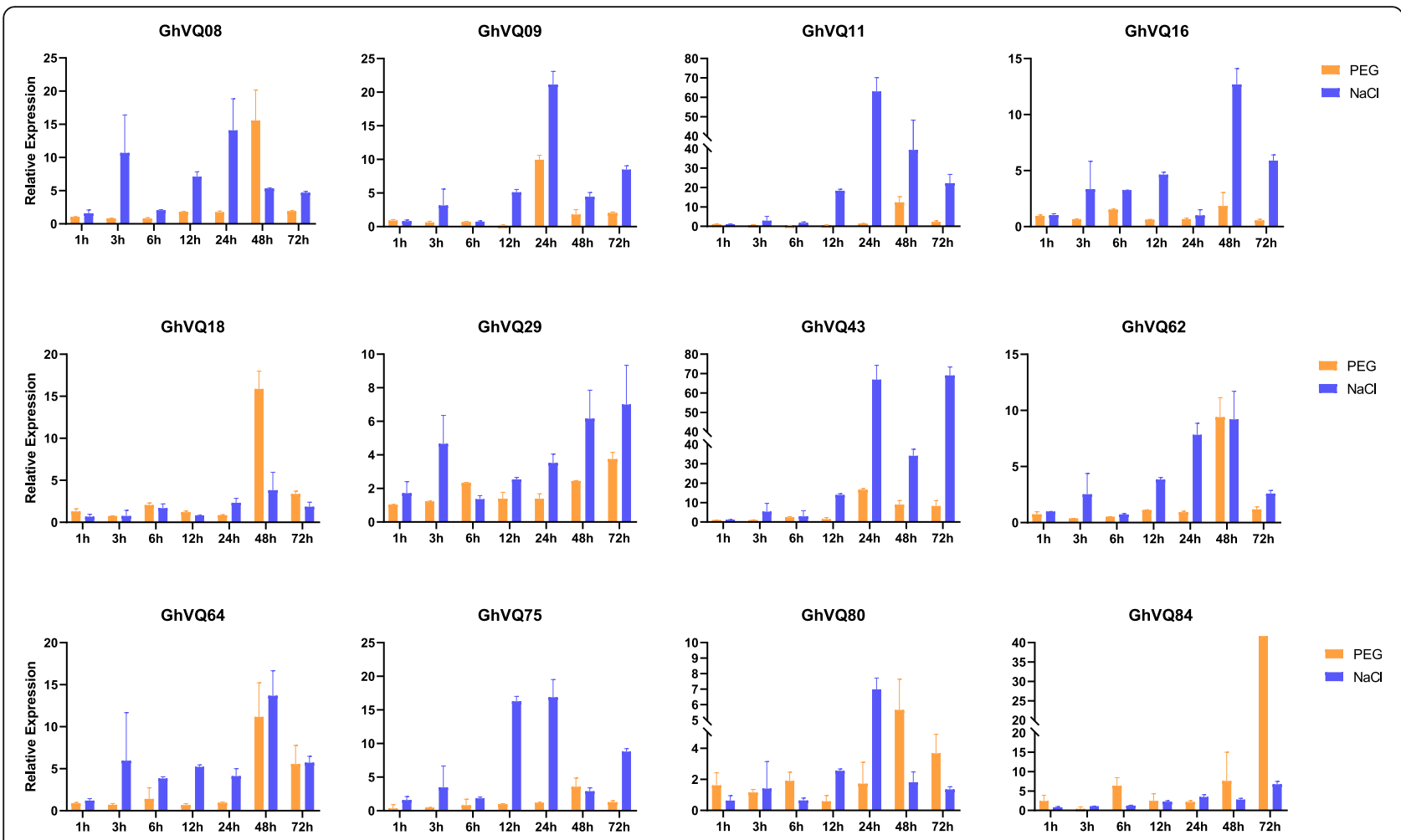

Fig. 9 The qPCR relative transcriptional levels of GhVQs under different stresses. The time of posts of different treatments are shown on the $x$-axis, and the relative expression levels are shown on the $y$-axis

of gene duplication. Collectively, our data speculated that Gossypium VQs might be affected by intronic evolution.

\section{VQs play important roles in abiotic stress signaling pathways}

Previous reports have shown that $V Q s$ are involved in various endogenous and environmental signals, which consistent with their diverse roles in plant development and in the response to abiotic stresses $[2,5,19,24,26$, 32]. For example, AtVQ08, AtVQ14, AtVQ17, AtVQ18 and $A t V Q 22$ are involved in modulation of seed development, chloroplast development, and plant growth. A proportion of the GmVQs [25], PeVQs [44], $V v V Q s$ [48], CsVQs [29], HaVQs [31], and $N t V Q s$ [19] also function in regulating the growth of different tissues at different developmental stages. In this study, most GhVQs and $G b V Q s$ were found to differentially expressed in the different tissues, including the ovule, fiber, anther, leaf, root, sepal and stem, suggesting that the $V Q s$ may play an important role in growth and development of Gossypium species (Fig. 7, Additional Files 6 and 7, Supplemental Fig. S6 and S7). Most $V Q s$ have been demonstrated to play important roles in responses to various abiotic stresses in plants $[2,3,19,27,30,32]$. In our work, we assessed the expression levels of the $G h V Q s$ and $G b V Q s$ under salt, drought, cold, and heat stresses, and found that the majority of the $V Q s$ were up-regulated under drought, salt, and cold stress, or down-regulated under heat stress. These findings were similar to those of previous reports in Arabidopsis [5], rice [26], maize [27] and cabbage [28]. Also, the promoters of the Gossypium VQs, many cis-elements that were reported to exist in other abiotic stresses responsive genes were detected (Fig. 3), implying that the $V Q s$ in Gossypium are likely involved in response to various abiotic stresses, and that the response mechanisms maybe complex and diverse.

$V Q s$ has been reported to interact with WRKY TFs and to regulate a variety of physiological and biochemical processes and abiotic stress responses $[6,9,11,49$, 50]. Here, by constructing co-expression and an Arabidopsis associated model, multiple Gossypium VQs, such as GhVQ37, GhVQ59, and GhVQ83, were predicted to interact with different WRKY TFs, implying that $V Q s$ act in stress tolerance through interacting with WRKYs. Moreover, we predicted some putative target sites of microRNAs in the Gossypium VQs. These microRNAs included miRNA156s and miR172, which serve important roles in various life processes of plants [40-42]. 


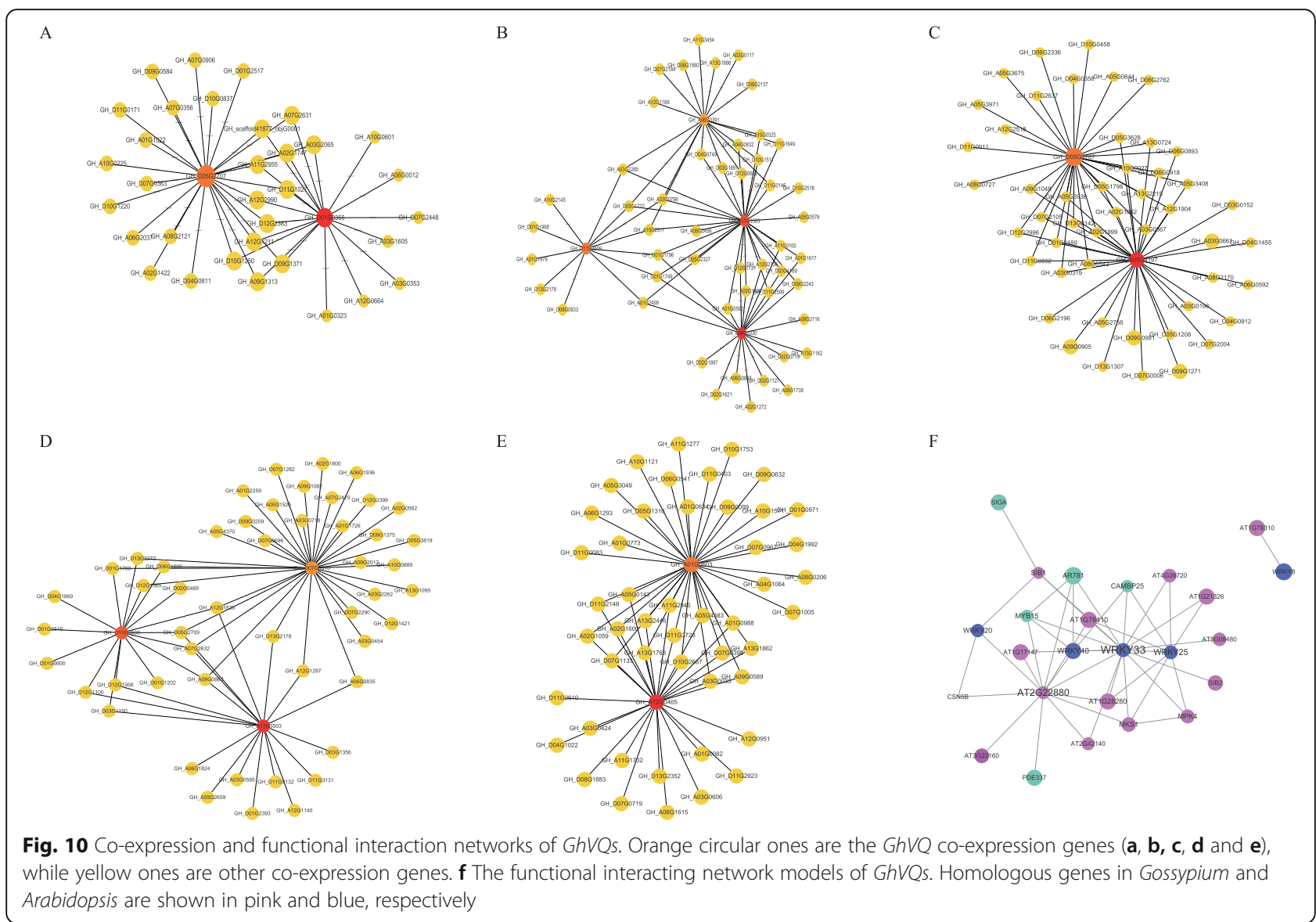

These results indicate that the Gossypium VQs are extensively involved in growth, development, and in response to stresses, and work together with WRKYs and microRNAs during these processes.

\section{Conclusions}

In this study, using bioinformatics plus expression profiles, we identified and presented the structure, phylogenetic relationships, and tissue specificity of $V Q$ family genes in four Gossypium species. Our data showed that the gene structure and motif coding regions were conserved across plants, and segmental, dispersed, and tandem duplications were the main reason for the expansion of the $V Q s$. Cis-element and expression analyses indicated that the majority of $V Q s$ were activated in response to abiotic stress, and some of $V Q s$ were coexpressed with WRKYs and hybridized with the miRNAs involved in Gossypium growth, development, and abiotic stress. Our study could serve as a foundation for future exploration of the specific functions of Gossypium $V Q s$ in the abiotic stress responses and the interactions with WRKYs or microRNAs.

\section{Methods}

Identification and classification of VQs in plants

The latest versions of predicted proteomes of G. raimondii (https://phytozome.jgi.doe.gov/pz/portal.html\#!info?alias=Org_Graimondii) [35], G. arboreum (ftp:// bioinfo.ayit.edu.cn/downloads/) [34], G. hirsutum, and G. barbadense (https://ibi.zju.edu.cn/cotton/) [33] were used in this study. The genome data of other plants were obtained from the JGI database (https://www.phytozome.net) and National Center for Biotechnology Information (NCBI) database (https://www.ncbi.nlm.nih.gov/ ): A. thanliana, $V$. vinifera, T. cacao, P. trichocarpa, $P$. dactylifera, O. sative, M. acuminate, D. zibethinus, C. papaya, B. rapacious, and $A$. trichopoda. The preclassified groups of these species were based on their phylogenetic relationships (https://www.timetree.org/) [51]. The VQ conserved domain (PF05678) was used as a query to scan the Gossypium species protein databases, and the $A$. thaliana VQ proteins were used as the queries to search against the above proteomes through the basic local alignment search tool (BLAST, $\mathrm{v} 2.10 .0$ ) (score value $\geq 0.0001$ and $E$-value $=1 \times 10^{-3}$ ) [52] for each newly identified gene. The obtained putative VQ 
motif-containing sequences were confirmed in the NCBI Conserved Domain Database (https://www.ncbi.nlm.nih. gov/Structure/cdd/wrpsb.cgi) [53] and SMART database (https://smart.embl-heidelberg.de/) [54]. Then, the physical and chemical properties of the $V Q$ family members, including amino acid length, mRNA length, MW, and pI, were analyzed using the online tools of the web site of softberry website (https://linux1.softberry.com/berry. phtml) and the ExPASy website (https://web.expasy.org/ translate/) [55], and the relative results were plotted by the ggstatsplot ( $\mathrm{v}$ 0.4.0) [56].

\section{Phylogenetic and synteny analysis of the VQs in plants}

All the VQ motif-containing proteins from the four Gossypium and other 11 plant species were aligned using MAFFT (Multiple Alignment using Fast Fourier Transform, v 7.4.0.7) (L-INS-algorithms) [57] with default parameters, and conserved site sequences were selected by the Gblock (v 0.91b) software (https://molevol.cmima. csic.es/castresana/Gblocks_server.html) [58]. A phylogenetic tree was constructed using the IQ-TREE software (v 1.6.9) (https://www.iqtree.org/) [59] with the maximum likelihood method, and the substitution model was calculated with ModelFinder (intergraded in IQ-TREE; best-fit model: JTT + R5 chosen according to $\mathrm{BIC})$. The obtained treefile was visualized using the ggtree (v 2.0.2) [60] and AI (Adobe Illustrator CS6).

The synteny and collinearity of duplication genes were analyzed in Gossypium species including G. hirsutum and G. arboreum, G. hirsutum, and G. raimondii; G. barbadense and G. arboreum; G. barbadense and G. raimondii; and G. hirsutum and G. barbadense using the modified MCScan algorithm of the MCScanX package (default parameters) (https://chibba.pgml.uga.edu/ mcscan2/) [61]. All results were drawn using Circos (https://circos.ca/) [62].

\section{Analysis of chromosome location and gene regulatory elements}

The chromosomal positions of all $V Q$ members were determined using the gene transfer (gtf) format files of the reference genomes. The exon/intron structure of VQs were also extracted from the gtf files and displayed by the GSDS platform (http://gsds.cbi.pku.edu.cn/) [63]. Then, the MEME tool (http://meme-suite.org/) (the motif with 10 amino acids in length and E-value less than $1 \mathrm{e}^{-40}$ ) [64] was used to detect the additional motifs of the proteins. With the combination of TBtools software (v 0.67361) [65], all domain motifs were compared among $V Q$ genes to identify the group-specific signatures.

Approximately $2000 \mathrm{bp}$ genomic sequences locating upstream (from - 2000 to -1 ) of the $V Q s$ from start codons were extracted from the cotton genome, which was subsequently submitted to the online PlantCARE (http:// bioinformatics.psb.ugent.be/webtools/plantcare/html/) [66] to determine the distribution of plant cis-acting regulatory elements. Moreover, the miRNA targets of the VQ members were predicted using the coding sequences (CDS) regions for complementary sequences by the psRNATarget server (http://plantgrn.noble.org/ psRNATarget/analysis?function=2) [67] with default parameters, except maximum expectation $(E)=3.5$. A total of 80 published miRNAs of G. hirsutum were selected.

\section{Expression profiles and co-expression networks}

To determine the expression patterns of $V Q s$ in the two allotetraploid kinds of Gossypium, RNA-Seq data were obtained from the SRA database (PRJNA490626) [33], including those in 10 tissues (the petal, pistil, root, sepal, stem, torus, filament, leaf, anther, and bract), during ovule development $(-3,-1,0,1,3,5,10,20$, and 25 DPA ovule) and fiber development (10, 20, and 25 DPA), and under four different abiotic stresses $(\mathrm{NaCl}$, PEG, cold, and hot), which were previously generated and analyzed by $\mathrm{Hu}$ et al. [33]. Raw RNA-seq reads were filtered using the SRAToolkit (v 2.9.2) / fastq-dump [68] and trimmed by Trimmomatic (v 0.3.9) [69] to generate clean reads. Then, the filtered clean RNA-seq reads were mapped to their respective reference genomes using the HISAT2 ( $v$ 2.1.0) [70], and the Sequence Alignment/ Map format (sam) data was converted to Binary Alignment/Map (bam) data using the samtools (v 1.9) [71]. The transcript abundances of annotated genes were quantified using the stringtie ( $v$ 2.0) [72]. The gene expression data were obtained from the big databases by $\mathrm{R}$ base, and expression levels expressed as $\log 2($ FPKM+1) and visualized using the pheatmap ( $\mathrm{v}$ 1.0.12) [73]. The WGCNA (v 1.69) [74] and the STRING software (Searched Tool for the Retrieval of Interacting Genes/ Proteins, http://string-db.org/) [75] were used for the analysis the co-expression networks, and the obtained results were displayed using the Cytoscape software $(\mathrm{v}$ 3.7.2) (http://www.cytoscape.org/) [76].

\section{Plant cultivation, RNA isolation, and RT-PCR analysis}

The germinated TM-1 cotton seeds were grown in plastic pots filled with the mixture of soil vermiculite, and the artificial growth conditions were set at $28 / 22^{\circ} \mathrm{C}$, with a photoperiod of $16 \mathrm{~h}$ light $/ 8 \mathrm{~h}$ darkness. Plants were separately subjected to $400 \mathrm{mM}$ PEG or $400 \mathrm{mM} \mathrm{NaCl}$. Three biological replicates were sampled at $0,1,3,6,12$, 24,36 , or $72 \mathrm{~h}$. All the samples were collected and frozen in liquid nitrogen, which was stored at $-80^{\circ} \mathrm{C}$ until total RNA extraction.

Total RNAs of the above samples were isolated using the RNA prep Pure Plant Kit (Polysaccharides \& Polyphenolics-rich, DP441) (TIANGEN, Beijing, China). 
The concentrations and integrities of the extracted RNA samples were measured and verified using a NanoDrop machine and 1\% agarose gel electrophoresis, and the RNA samples were reversed transcribed into complementary DNA (cDNA) using the Mir-X TM MIRNA First-Strand Synthesis Kit (TaKaRa, Dalian, China). The qRT-PCR was performed using the Roche LightCycler 480 System (Roche, Germany). The qRT-PCR primers for the GhVQs and actin gene were listed in Supplemental Table S8. The reaction was set up in a total volume of $20 \mu \mathrm{L}: 2 \mu \mathrm{L}(200 \mathrm{ng})$ of cDNA, $0.4 \mu \mathrm{L}$ of forwarding primer $(10.0 \mu \mathrm{M}), 0.4 \mu \mathrm{L}$ of reverse primer $(10.0 \mu \mathrm{M})$, $10 \mu \mathrm{L} 2 \times$ TransStart Top/Tip Green qPCR SuperMix, and $7.2 \mu \mathrm{L}$ of nuclease-free water. The reaction procedure was completed under the following program: $94{ }^{\circ} \mathrm{C}$ for $30 \mathrm{~s} ; 45$ cycles of $94{ }^{\circ} \mathrm{C}$ for $5 \mathrm{~s}, 60^{\circ} \mathrm{C}$ for $15 \mathrm{~s}, 72^{\circ} \mathrm{C}$ for $10 \mathrm{~s}$; and $4{ }^{\circ} \mathrm{C}$ to finish. The results were calculated using the $2^{-\Delta \Delta \mathrm{Ct}}$ relative quantitative method.

\section{Supplementary Information}

Supplementary information accompanies this paper at https://doi.org/10. 1186/s12864-020-07171-z.

Additional file 1: Table S1. Summaries of VQ genes members in Gossypium

Additional file 2: Figure S1. The physical and chemical properties of VQs in Gossypium spp. with violin illustration. $\mathbf{a}, \mathbf{b}, \mathbf{c}, \mathbf{d}, \mathbf{e}, \mathbf{f}$ are the lengths of the transcripts, GC contents, exon numbers, protein lengths, PI values and MW values, respectively.

Additional file 3: Table S2. The number of $V Q$ genes in the 15 plant genomes.

Additional file 4: Figure S2. The phylogenetic tree of VQs in Gossypium spp. compared with A. thaliana. a The phylogenetic tree of $V Q$ s in G. hirsutum and A. thaliana. $\mathbf{b}$ The phylogenetic tree of VQs in G. barbadense and $A$. thaliana. c The phylogenetic tree of VQs in G. raimondii and $A$. thaliana. d The phylogenetic tree of VQs in G. arboretum and $A$. thaliana.

Additional file 5: Figure S3. The conserved motifs of VQs.

Additional file 6: Figure S4. The paralogs of VQs between G. hirsutum and another two diploid Gossypium species. The lines regarding orthologous gene pairs are colored by green and blue. The green lines are the pairs between G. hirsutum D-subgenome and G. raimondii genome, and the blue lines are the pairs between $G$. hirsutum A-subgenome and $G$. arboretum genome.

Additional file 7: Table S3. The list of paralogous VQ gene pairs in each of other examined species.

Additional file 8: Figure S5. The paralogs of VQs between G. barbadense and another two diploid Gossypium species. The lines regarding orthologous gene pairs are colored by green and blue. The green lines are the pairs between $G$. barbadense D-subgenome and G. raimondii genome; and the blue lines are the pairs between $G$. barbadense A-subgenome and $G$. arboretum genome.

Additional file 9: Table S4. The duplicated type of VQs in Gossypium spp. The 0 to 4 indicate the singleton, dispersed, proximal, tandem, WGD duplication types, respectively.

Additional file 10: Table S5. List of predicted known microRNA target sites of VQ transcripts in Gossypium species.

Additional file 11: Figure S6. Expression patterns of GhVQs in different tissues and under stresses in G. hirsutum.
Additional file 12: Figure S7. Expression patterns of GbVQs in different tissues and under stresses in G. barbadense.

Additional file 13: Table S6. Expression levels of GhVQs and GbVQs. The gene expression was determined by RNA-Seq data (FPKM).

Additional file 14: Table S7. Information regarding the Co-expression and STRING search results of the interaction networks of the GhVQs.

Additional file 15: Table S8. Primers used for $q P C R$ analyses of GhVQs.

Acknowledgements

We are grateful to all the colleagues in our laboratory.

\section{Authors' contributions}

S.X.Y. and H.L.W. designed the research program. F.W., S.S.C., L.M. and H.T.W. analyzed and transcriptomic data. M.Z., G.Z.M., J.H.L., P.B.H., and A.M.W. participated in cotton culture processing and RNA extraction, A.A., L.J.G., and Q.M. revised the language. P.Y.C. performed the experiment and wrote the manuscript. All authors have read and approved the final manuscript.

\section{Funding}

This project is based on research that was supported by Central Publicinterest Scientific Institution Basal Research Fund (No. Y2018YJ05).

Availability of data and materials

All data supporting the conclusions of this article are included in the article and its additional files.

Ethics approval and consent to participate

Not applicable.

Consent for publication

Not applicable.

\section{Competing interests}

The authors declare that they have no competing interests.

\section{Author details}

${ }^{1}$ State Key Laboratory of Cotton Biology, Institute of Cotton Research of Chinese Academy of Agricultural Sciences, Anyang 455000, Henan, China. ${ }^{2}$ School of Life Science, Zhengzhou University, Zhengzhou 450000, China. ${ }^{3}$ College of Agronomy, Northwest A\&F University, Yangling 712100, China.

Received: 23 June 2020 Accepted: 21 October 2020

Published online: 16 November 2020

\section{References}

1. Wang A, Garcia D, Zhang H, Feng K, Chaudhury A, Berger F, Peacock WJ, Dennis ES, Luo M. The VQ motif protein IKU1 regulates endosperm growth and seed size in Arabidopsis. Plant J. 2010;63(4):670-9.

2. Jiang SY, Sevugan M, Ramachandran S. Valine-glutamine (VQ) motif coding genes are ancient and non-plant-specific with comprehensive expression regulation by various biotic and abiotic stresses. BMC Genomics. 2018;19(1):342.

3. Cai HY, Zhang M, Liu YH, He Q, Chai MN, Liu LP, Chen FQ, Huang YM, Yan MK, Zhao HM, et al. Genome-wide classification and evolutionary and functional analyses of the VQ family. Trop Plant Biol. 2019;12(2):117-31.

4. Xie YD, Li W, Guo D, Dong J, Zhang Q, Fu Y, Ren D, Peng M, Xia Y. The Arabidopsis gene sigma factor-binding protein 1 plays a role in the salicylate- and jasmonate-mediated defence responses. Plant Cell Environ. 2010;33(5):828-39.

5. Jing $Y$, Lin $R$. The VQ motif-containing protein family of plant-specific transcriptional regulators. Plant Physiol. 2015;169(1):371-8.

6. Lai Z, Li Y, Wang F, Cheng Y, Fan B, Yu JQ, Chen Z. Arabidopsis sigma factor binding proteins are activators of the WRKY33 transcription factor in plant defense. Plant Cell. 2011:23(10):3824-41.

7. Jiang Y, Yu D. The WRKY57 transcription factor affects the expression of Jasmonate ZIM-domain genes transcriptionally to compromise Botrytis cinerea resistance. Plant Physiol. 2016;171(4):2771-82.

8. Hu P, Zhou W, Cheng Z, Fan M, Wang L, Xie D. JAV1 controls jasmonateregulated plant defense. Mol Cell. 2013;50(4):504-15. 
9. Hu Y, Chen L, Wang H, Zhang L, Wang F, Yu D. Arabidopsis transcription factor WRKY8 functions antagonistically with its interacting partner VQ9 to modulate salinity stress tolerance. Plant J. 2013;74(5):730-45.

10. Dong Q, Zhao S, Duan D, Tian Y, Wang Y, Mao K, Zhou Z, Ma F. Structural and functional analyses of genes encoding VQ proteins in apple. Plant Sci. 2018;272:208-19.

11. Xixu P, Ting X, Jiao M, Zong T, Ding-gang Z, Xin-ke T, Hai-hua W. Differential expression of Rice Valine-Qlutamine gene family in response to nitric oxide and regulatory circuit of OsVQ7 and OsWRKY24. Rice Sci. 2019;27:10-20.

12. Lei R, Li X, Ma Z, Lv Y, Hu Y, Yu D. Arabidopsis WRKY 2 and WRKY 34 transcription factors interact with VQ 20 protein to modulate pollen development and function. Plant J. 2017;91(6):962-76.

13. Pecher P, Eschen-Lippold L, Herklotz S, Kuhle K, Naumann K, Bethke G, Uhrig J, Weyhe M, Scheel D, Lee J. The Arabidopsis thaliana mitogenactivated protein kinases MPK3 and MPK6 target a subclass of 'VQ-motif'containing proteins to regulate immune responses. New Phytol. 2014;203(2): 592-606.

14. Xie F, Wang Q, Sun R, Zhang B. Deep sequencing reveals important roles of microRNAs in response to drought and salinity stress in cotton. J Exp Bot. 2015;66(3):789-804.

15. Eulgem T, Rushton PJ, Robatzek S, Somssich IE. The WRKY superfamily of plant transcription factors. Trends Plant Sci. 2000;5(5):199-206.

16. Dou L, Zhang X, Pang C, Song M, Wei H, Fan S, Yu S. Genome-wide analysis of the WRKY gene family in cotton. Mol Gen Genomics. 2014;289(6):1103-21.

17. Grandbastien MA. LTR retrotransposons, handy hitchhikers of plant regulation and stress response. Biochim Biophys Acta. 2015;1849(4):403-16.

18. Zhu JK. Plant salt tolerance. Trends Plant Sci. 2001;6(2):66-71.

19. Liu C, Liu H, Zhou C, Timko MP. Genome-Wide Identification of the VQ Protein Gene Family of Tobacco (Nicotiana tabacum L.) and Analysis of Its Expression in Response to Phytohormones and Abiotic and Biotic Stresses. Genes (Basel). 2020;11(3):284

20. Roberts EM, Rao NR, Huang JY, Trolinder NL, Haigler CH. Effects of cycling temperatures on fiber metabolism in cultured cotton ovules. Plant Physiol. 1992:100(2):979-86.

21. Khan A, Pan X, Najeeb U, Tan DKY, Fahad S, Zahoor R, Luo H. Coping with drought: stress and adaptive mechanisms, and management through cultural and molecular alternatives in cotton as vital constituents for plant stress resilience and fitness. Biol Res. 2018:51(1):47.

22. Wang W, Vinocur B, Altman A. Plant responses to drought, salinity and extreme temperatures: towards genetic engineering for stress tolerance. Planta. 2003;218(1):1-14

23. Cramer GR, Urano K, Delrot S, Pezzotti M, Shinozaki K. Effects of abiotic stress on plants: a systems biology perspective. BMC Plant Biol. 2011; 11(1):163.

24. Li Y, Jing Y, Li J, Xu G, Lin R. Arabidopsis VQ motif-containing protein29 represses seedling deetiolation by interacting with phytochrome-interacting factor1. Plant Physiol. 2014;164(4):2068-80.

25. Wang Y, Jiang Z, Li Z, Zhao Y, Tan W, Liu Z, Cui S, Yu X, Ma J, Wang G, et al. Genome-wide identification and expression analysis of the VQ gene family in soybean (Glycine max). PeerJ. 2019;7:e7509.

26. Kim DY, Kwon SI, Choi C, Lee H, Ahn I, Park SR, Bae SC, Lee SC, Hwang DJ. Expression analysis of rice $\mathrm{VQ}$ genes in response to biotic and abiotic stresses. Gene. 2013;529(2):208-14.

27. Song W, Zhao H, Zhang X, Lei L, Lai J. Genome-wide identification of VQ motif-containing proteins and their expression profiles under abiotic stresses in maize. Front Plant Sci. 2016;6:1177.

28. Zhang G, Wang F, Li J, Ding Q, Zhang Y, Li H, Zhang J, Gao J. Genome-wide identification and analysis of the VQ motif-containing protein family in Chinese cabbage (Brassica rapa L. ssp. Pekinensis). Int J Mol Sci. 2015;16(12): 28683-704.

29. Guo J, Chen J, Yang J, Yu Y, Yang Y, Wang W. Identification, characterization and expression analysis of the VQ motif-containing gene family in tea plant (Camellia sinensis). BMC Genomics. 2018;19(1):710.

30. Ding H, Yuan G, Mo S, Qian Y, Wu Y, Chen Q, Xu X, Wu X, Ge C. Genomewide analysis of the plant-specific $V Q$ motif-containing proteins in tomato (Solanum lycopersicum) and characterization of SIVQ6 in thermotolerance. Plant Physiol Biochem. 2019;143:29-39.

31. Ma J, Ling L, Huang X, Wang W, Yb W, Zhang M, Sy C, Zhou F, Yk Q, Cb L, et al. Genome-wide identification and expression analysis of the VQ gene family in sunflower (Helianthus annuus L.). J Plant Biochem Biotechnol. 2020:1-11. https://doi.org/10.1007/s13562-020-00568-7.
32. Ling L, Qu Y, Zhu J, Wang D, Guo C. Genome-wide identification and expression analysis of the VQ gene family in Cicer arietinum and Medicago truncatula. PeerJ. 2020;8:e8471.

33. Hu Y, Chen J, Fang L, Zhang Z, Ma W, Niu Y, Ju L, Deng J, Zhao T, Lian J, et al. Gossypium barbadense and Gossypium hirsutum genomes provide insights into the origin and evolution of allotetraploid cotton. Nat Genet. 2019;51(4):739-48

34. Du X, Huang G, He S, Yang Z, Sun G, Ma X, Li N, Zhang X, Sun J, Liu M, et al. Resequencing of 243 diploid cotton accessions based on an updated a genome identifies the genetic basis of key agronomic traits. Nat Genet. 2018;50(6):796-802.

35. Paterson AH, Wendel JF, Gundlach $H$, Guo H, Jenkins J, Jin D, Llewellyn D, Showmaker KC, Shu S, Udall J, et al. Repeated polyploidization of Gossypium genomes and the evolution of spinnable cotton fibres. Nature. 2012:492(7429):423-7.

36. Lee TH, Tang H, Wang X, Paterson AH. PGDD: a database of gene and genome duplication in plants. Nucleic Acids Res. 2012:41(D1):D1152-8.

37. Wang H, Hu Y, Pan J, Yu D. Arabidopsis VQ motif-containing proteins VQ12 and VQ29 negatively modulate basal defense against Botrytis cinerea. Sci Rep. 2015;5(1):14185

38. Pan JJ, Wang HP, Hu Y, Yu D. Arabidopsis VQ18 and VQ26 proteins interact with $A B \mid 5$ transcription factor to negatively modulate $A B A$ response during seed germination. Plant J. 2018;95(3):529-44.

39. Zhang Y, Zhu X, Chen X, Song C, Zou Z, Wang Y, Wang M, Fang W, Li X. Identification and characterization of cold-responsive microRNAs in tea plant (Camellia sinensis) and their targets using high-throughput sequencing and degradome analysis. BMC Plant Biol. 2014;14(1):271.

40. Wu G, Park MY, Conway SR, Wang JW, Weigel D, Poethig RS. The sequential action of miR156 and miR172 regulates developmental timing in Arabidopsis. Cell. 2009;138(4):750-9.

41. Naqvi AR, Haq QM, Mukherjee SK. MicroRNA profiling of tomato leaf curl New Delhi virus (tolcndv) infected tomato leaves indicates that deregulation of mir159/319 and mir172 might be linked with leaf curl disease. Virol J. 2010;7(1):281

42. Stief A, Altmann S, Hoffmann K, Pant BD, Scheible WR, Baurle I. Arabidopsis miR156 regulates tolerance to recurring environmental stress through SPL transcription factors. Plant Cell. 2014;26(4):1792-807.

43. Ye YJ, Xiao YY, Han YC, Shan W, Fan ZQ, Xu QG, Kuang JF, Lu WJ, Lakshmanan P, Chen JY. Banana fruit VQ motif-containing protein5 represses cold-responsive transcription factor MaWRKY26 involved in the regulation of JA biosynthetic genes. Sci Rep. 2016;6(1):23632

44. Wang Y, Liu H, Zhu D, Gao Y, Yan H, Xiang Y. Genome-wide analysis of VQ motif-containing proteins in Moso bamboo (Phyllostachys edulis). Planta. 2017;246(1):165-81.

45. Adams KL, Wendel JF. Polyploidy and genome evolution in plants. Curr Opin Plant Biol. 2005;8(2):135-41.

46. Fawcett JA, Maere S, Van de Peer Y. Plants with double genomes might have had a better chance to survive the cretaceous-tertiary extinction event. Proc Natl Acad Sci U S A. 2009:106(14):5737-42.

47. Pellicer J, Hidalgo O, Dodsworth S, Leitch IJ. Genome size diversity and its impact on the evolution of land plants. Genes (Basel). 2018;9(2):88.

48. Wang M, Vannozzi A, Wang G, Zhong Y, Corso M, Cavallini E, Cheng ZM. A comprehensive survey of the grapevine VQ gene family and its transcriptional correlation with WRKY proteins. Front Plant Sci. 2015;6:417.

49. Cheng $Y$, Zhou Y, Yang Y, Chi YJ, Zhou J, Chen JY, Wang F, Fan B, Shi K, Zhou $Y H$, et al. Structural and functional analysis of VQ motif-containing proteins in Arabidopsis as interacting proteins of WRKY transcription factors. Plant Physiol. 2012;159(2):810-25

50. Perruc E, Charpenteau M, Ramirez BC, Jauneau A, Galaud JP, Ranjeva R, Ranty B. A novel calmodulin-binding protein functions as a negative regulator of osmotic stress tolerance in Arabidopsis thaliana seedlings. Plant J. 2004:38(3):410-20

51. Kumar S, Stecher G, Suleski M, Hedges SB. TimeTree: a resource for timelines, Timetrees, and divergence times. Mol Biol Evol. 2017;34(7):1812-9.

52. Altschul SF. Basic local alignment search tool (BLAST). J Mol Biol. 1990; 215(3):403-10.

53. Marchler-Bauer A, Lu S, Anderson JB, Chitsaz F, Derbyshire MK, Carol D-S, Fong JH, Geer LY, Geer RC, Gonzales NR. CDD: a conserved domain database for the functional annotation of proteins. Nucleic Acids Res. 2010;39:225-9.

54. Ivica L, Tobias D, Peer B. SMART 6: recent updates and new developments. Nucleic Acids Res. 2009;37:229-32. 
55. Panu A, Manohar J, Konstantin A, Delphine B, Gabor C, Edouard DC, Séverine D, Volker F, Arnaud F, Elisabeth G. ExPASy: SIB bioinformatics resource portal. Nucleic Acids Res. 2012;40:597-603.

56. Wickham H. ggplot2: elegant graphics for data analysis. New York: Springer; 2009. http://had.co.nz/ggplot2/book.

57. Katoh K, Standley DM. MAFFT: iterative refinement and additional methods. Methods Mol Biol. 2014;1079(1079):131-46.

58. Talavera G, Castresana J. Improvement of phylogenies after removing divergent and ambiguously aligned blocks from protein sequence alignments. Syst Biol. 2007;56(4):564-77.

59. Nguyen L, Schmidt H, Haeseler AV, Minh B. IQ-TREE: a fast and effective stochastic algorithm for estimating maximum-likelihood phylogenies. Mol Biol Evol. 2015;1:268-74.

60. Yu GC, Smith DK, Hc Z, Guan Y, Lam TT, Mclnerny G. GGtree : an r package for visualization and annotation of phylogenetic trees with their covariates and other associated data. Methods Ecol Evol. 2016;8(1):28-36.

61. Wang Y, Tang H, Debarry JD, Tan X, Li J, Wang X, Lee TH, Jin H, Marler B, Guo H, et al. MCScanX: a toolkit for detection and evolutionary analysis of gene synteny and collinearity. Nucleic Acids Res. 2012;40(7):e49.

62. Krzywinski M, Schein J, Birol I, Connors J, Gascoyne R, Horsman D, Jones SJ, Marra MA. Circos: an information aesthetic for comparative genomics. Genome Res. 2009;19(9):1639-45.

63. Hu B, Jin J, Guo AY, Zhang H, Luo J, Gao G. GSDS 2.0: an upgraded gene feature visualization server. Bioinformatics. 2015;31(8):1296-7.

64. Bailey TL, Boden M, Buske FA, Frith M, Grant CE, Clementi L, Ren J, Li WW, Noble WS. MEME SUITE: tools for motif discovery and searching. Nucleic Acids Res. 2009;37:W202-8.

65. Chen C, Chen H, Zhang Y, Thomas HR, Frank MH, He Y, Xia R. TBtools: an integrative toolkit developed for interactive analyses of big biological data. Mol Plant. 2020;13:1194-202.

66. Lescot M, Dehais P, Thijs G, Marchal K, Moreau Y, Van de Peer Y, Rouze P, Rombauts S. PlantCARE, a database of plant cis-acting regulatory elements and a portal to tools for in silico analysis of promoter sequences. Nucleic Acids Res. 2002;30(1):325-7.

67. Dai X, Zhao PX. psRNATarget: a plant small RNA target analysis server Nucleic Acids Res. 2011;39:W155-9.

68. Leinonen R, Sugawara H, Shumway M. International nucleotide sequence database C: the sequence read archive. Nucleic Acids Res. 2011;39(Database issue):D19-21.

69. Bolger AM, Lohse M, Usadel B. Trimmomatic: a flexible trimmer for Illumina sequence data. Bioinformatics. 2014;30(15):2114-20

70. Kim D, Langmead B, Salzberg SL. HISAT: a fast spliced aligner with low memory requirements. Nat Methods. 2015;12(4):357-60.

71. Li H, Handsaker B, Wysoker A, Fennell T, Ruan J, Homer N, Marth G, Abecasis $G$, Durbin R. Genome project data processing S: the sequence alignment/ map format and SAMtools. Bioinformatics. 2009;25(16):2078-9.

72. Pertea M, Pertea GM, Antonescu CM, Chang TC, Mendell JT, Salzberg SL. StringTie enables improved reconstruction of a transcriptome from RNA-sea reads. Nat Biotechnol. 2015;33(3):290-5.

73. Kolde R, Kolde MR: Package 'pheatmap'. Bioconductor 1-6. Available at: https://CRAN.R-project.org/package=pheatmap. 2012.

74. Langfelder P, Horvath S. WGCNA: an R package for weighted correlation network analysis. BMC Bioinform. 2008;9(1):559.

75. Wagner R, Fischer M. The string-to-string correction problem. J ACM (JACM). 1974:21(1):168-73.

76. Shannon P, Markiel A, Ozier O, Baliga NS, Wang JT, Ramage D, Amin N, Schwikowski B, Ideker T. Cytoscape: a software environment for integrated models of biomolecular interaction networks. Genome Res. 2003;13(11): 2498-504.

\section{Publisher's Note}

Springer Nature remains neutral with regard to jurisdictional claims in published maps and institutional affiliations.

Ready to submit your research? Choose BMC and benefit from:

- fast, convenient online submission

- thorough peer review by experienced researchers in your field

- rapid publication on acceptance

- support for research data, including large and complex data types

- gold Open Access which fosters wider collaboration and increased citations

- maximum visibility for your research: over $100 \mathrm{M}$ website views per year

At $\mathrm{BMC}$, research is always in progress.

Learn more biomedcentral.com/submissions 